\title{
Working classification of chronic myeloproliferative disorders based on histological, haematological, and clinical findings
}

\author{
R BURKHARDT,$\dagger$ R BARTL,${ }^{*} \dagger$ K JÄGER,${ }^{*}$ B FRISCH, $\ddagger$ G KETTNER, $\dagger$ G MAHL, $\dagger$ \\ M SUND§
}

From the *Abteilung für Knochenmarksdiagnostik an der Medizinischen Klinik Innenstadt der Universität, Munich, West Germany, the †Arbeitsgruppe Hämatomorphologie des Instituts für Hämatologie, Gesellschaft für Strahlen- und Umweltforschung mbH (National Center for Environmental Sciences), Munich, West Germany, the $\ddagger$ Institute of Haematology, Tel-Aviv Municipal-Governmental Medical Centre, Sackler School of Medicine, Tel-Aviv University, Israel, and the §Institut für Medizinische Informatik und Systemforschung der Gesellschaft für Strahlen- und Umeltforschung mbH, (National Center for Environmental Sciences), Neuherberg, West Germany

SUMMARY Bone marrow biopsies of 850 patients with chronic myeloproliferative disorders were taken at initial diagnosis; and 169 sequential biopsies over periods of one to 188 months. Three micron sections of all biopsies were evaluated semiquantitatively with reference to the proliferating cell lines, anomalies of megakaryocytes, and fibrosis or osteosclerosis. Correlations between initial histological findings, clinical, haematological, and survival data were analysed statistically.

The predominant cell lines distinguished the classical entities of polycythaemia vera, primary thrombocythaemia, and chronic myeloid leukaemia and correlated with their different prognoses, while megakaryocytes characterised subgroups that were prone to fibrotic or blastic transformation. Based on the initial histological, clinical, and haematological data analysed a working classification of chronic myeloproliferative disorders was proposed that permits recognition of both typical and atypical cases of chronic myeloproliferative disorders.

Chronic myeloproliferative disorders are polycythaemia vera, primary thrombocythaemia, and chronic myeloid (or granulocytic) leukaemia. ${ }^{1}$ These groups are characterised by clinical, haematological, cytogenetic, and histological criteria. ${ }^{2-3}$ These criteria and therefore the groups, however, sometimes overlap or transform to more immature stages (blast crisis) or fibrotic stages, such as myelofibrosis, osteomyelosclerosis, or agnogenic myeloid metaplasia. ${ }^{4}$ The origin of agnogenic myeloid metaplasia from haemopoietic stem cells and the secondary nature of the fibrosis form the basis of previous pathogenetic classifications. ${ }^{5}$ The problem remains to coordinate the clinical aspects with the histological findings in the bone marrow. To this end untreated cases of histologically confirmed chronic myeloproliferative disorders were selected, irrespective of the clinical diagnosis, for retrospective

Accepted for publication 9 October 1985 analysis of initial bone marrow histology and clinical data, including statistical evaluation of correlations and prognostic importance, and of sequential biopsies obtained in some of the cases.

\section{Material and methods}

The biopsies of 850 patients, taken to clarify clinically suspected or diagnosed chronic myeloproliferative disorders, fulfilled the following criteria: the biopsy was of adequate quality; sufficient clinical data were available for diagnosis; diagnosis of chronic myeloproliferative disorders was possible according to histological or clinical criteria, or both; no treatment was given before initial bone biopsy. One hundred and twenty six of the patients underwent 169 sequential biopsies.

The biopsies were taken from the anterior or posterior iliac crests; minimal sizes $4 \times 10 \mathrm{~mm}^{2}$ (myelotomy drill, 781 cases) and $2 \times 20 \mathrm{~mm}^{2}$ (manual 
Table 1 Clinical variables (at time of biopsy)

\begin{tabular}{|c|c|}
\hline $\begin{array}{l}\text { Age } \\
\text { Sex } \\
\text { Clinical diagnosis } \\
\text { History of chronic } \\
\text { myeloproliferative disorders }\end{array}$ & $\begin{array}{l}\text { Haemoglobin } \\
\text { Erythrocyte count } \\
\text { Leucocyte count } \\
\text { Platelet count }\end{array}$ \\
\hline $\begin{array}{l}\text { Time from first symptom to } \\
\text { biopsy } \\
\text { Spleen size }\end{array}$ & $\begin{array}{l}\text { Blood smear, leucocyte alkaline } \\
\text { phosphatase score } \\
\text { Red blood cell erythrocyte } \\
\text { sedimentation rate }\end{array}$ \\
\hline Liver size & $\begin{array}{l}\text { Serum: iron, alkaline } \\
\text { phosphatase, lactate } \\
\text { dehydrogenase }\end{array}$ \\
\hline
\end{tabular}

trephine, 69 cases). All biopsies were fixed, embedded in plastic, and stained as previously described. ${ }^{6}$ In some cases small pieces of the biopsies were also processed for electron microscopy.

\section{CLINICAL EVALUATION}

Table 1 lists the clinical variables registered at diagnosis. These initial haematological values were used to establish the haematological groups. Survival data over periods of one to 160 months were available for 470 patients.

\section{HISTOLOGICAL EVALUATION}

All biopsies were studied by at least two independent investigators. Each biopsy was reviewed five times, and twenty two histological criteria were registered (Table 2). Both clinical and histological data were used for computer based multivariate analysis (BMDP programs). ${ }^{7}$ Histological data from previous quantitative evaluations of 158 biopsies of subjects with haematological and biochemical values within normal limits (mean age 38 years, range 2-92 years; $59 \%$ men, $41 \%$ women), served as baseline data. The presence or absence, increase or decrease of each variable was noted in each of the chronic myeloproliferative disorder biopsies studied. All correlations were statistically checked using student's $t$ test in most cases, the Pearson $\chi^{2}$ test, and the Fisher test according to Breslow and Mantel-Cox. ${ }^{7}$

\section{CRITERIA FOR PRELIMINARY CLASSIFICATION} la Polycythaemia vera (144 cases) Clinical data: red cell counts $>6.5 \times 10^{12} / 1$; typical clinical history of at least six months, erythrocyte sedimentation rate below $4 \mathrm{~mm} /$ hour, and exclusion of secondary erythrocytosis. Additional relevant data such as oxygen saturation of the blood, blood volume, leucocyte alkaline phosphatase, and chromosomal analysis were included when available. Bone biopsy data: erythrocytopoiesis, increased granulocytopoiesis and megakaryocytopoiesis; sinusoidal hyperplasia; reduction of fatty tissue; greatly reduced iron storage.

lb Polycythaemia vera borderline (50 cases) Either clinical data as above, but with a less typical histological picture, or histology as above with clinical criteria as above, except for red cell counts of $5 \cdot 5-6.5 \times 10^{12} / 1$.

2a Chronic myeloid leukaemia (108 cases) Clinical data: leucocyte count $>30 \times 10^{9} / 1$, typical blood smear, splenic enlargement (most cases), and exclusion of secondary leucocytosis. Additional relevant data such as measurements of leucocyte alkaline phosphatase and chromosomal analysis were included when available. Bone biopsy data: increased granulocytopoiesis; decreased erythrocytopoiesis and fatty tissue; decrease or increase in normal or small megakaryocytes.

2b Chronic myeloid leukaemia borderline (17 cases) Clinical data: leucocyte count 15-30 × 109/1; rest of clinical and biopsy criteria as above.

3a Primary thrombocythaemia (27 cases) Clinical data: platelet count $>700 \times 10^{9} / 1$, and exclusion of secondary thrombocythaemia. Additional relevant data, such as measurements of leucocyte alkaline phosphatase and chromosomal analysis were included when available. Bone biopsy data: increased megakaryocytes; numerous giant forms; and decreased, normal, or slightly increased erythrocytopoiesis and granulocytopoiesis.

3b Primary thrombocythaemia borderline (16 cases) Clinical data: platelet count $300-700 \times 10^{9} / 1$; rest of clinical and biopsy criteria as above.

4 Myelofibrosis (78 cases) Bone biopsy data: presence of numerous strands of coarse fibres, enclosing areas of hypercellular marrow containing

Table 2 Histological variables (iliac crest biopsy)

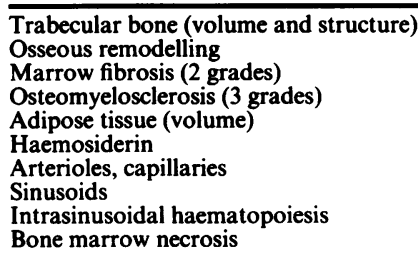

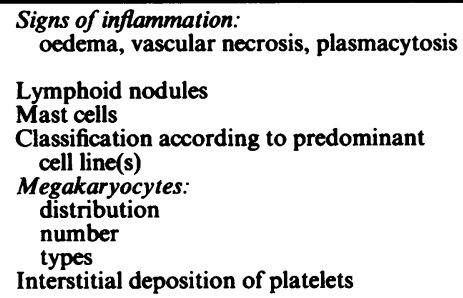

Interstitial deposition of platelets 

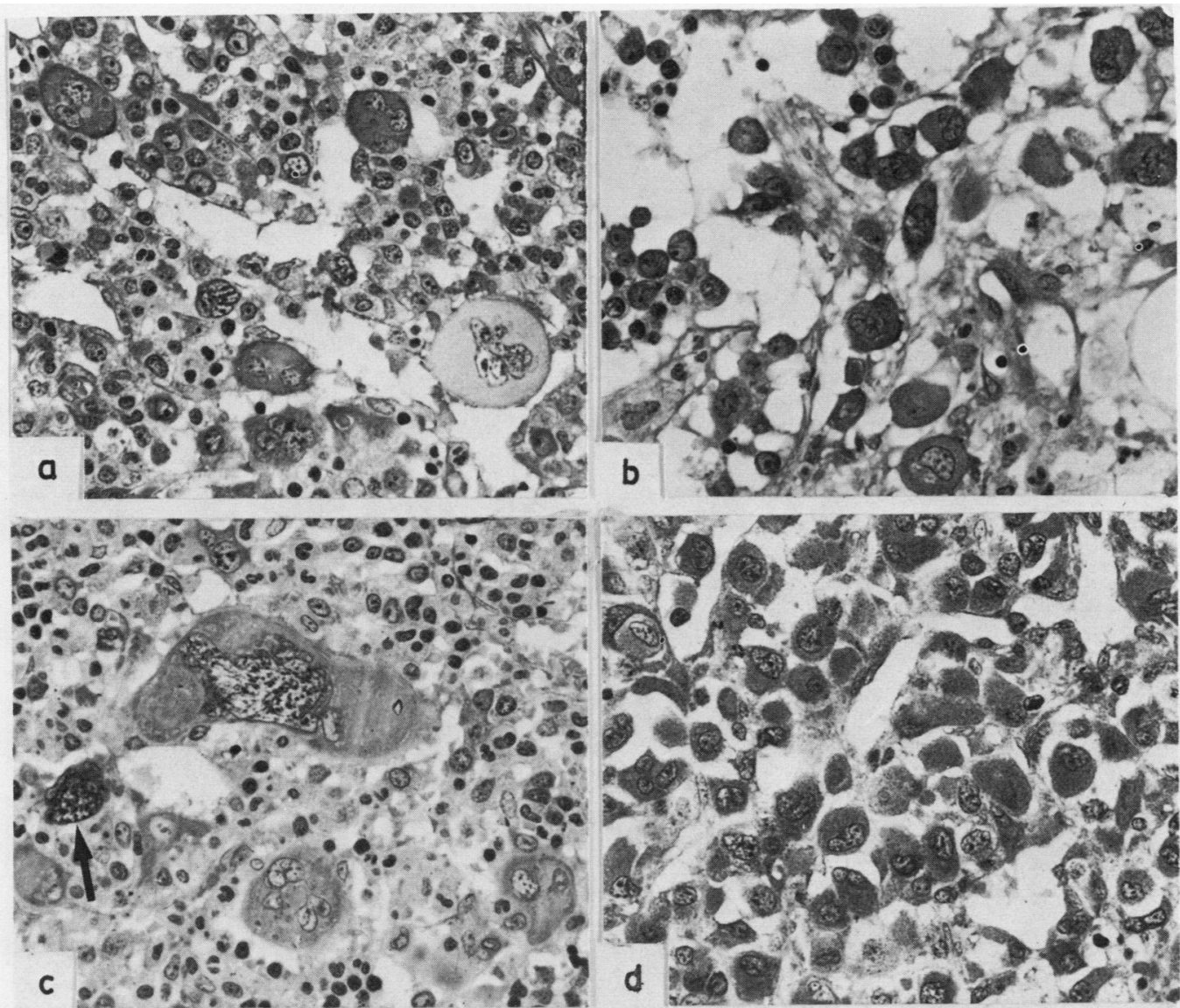

2.

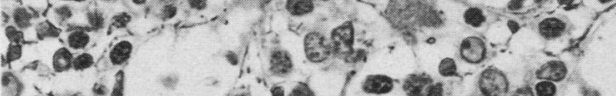

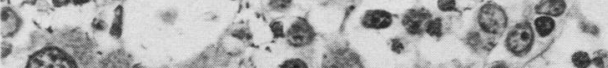
(3)

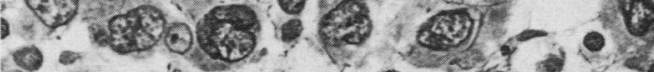

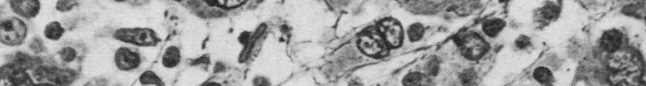
3.9.

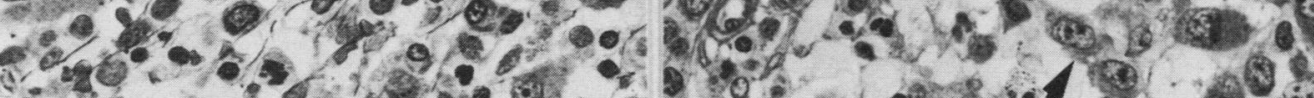

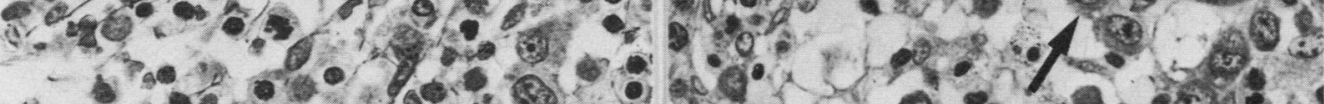

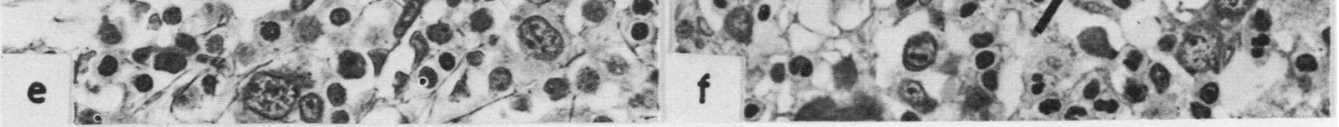

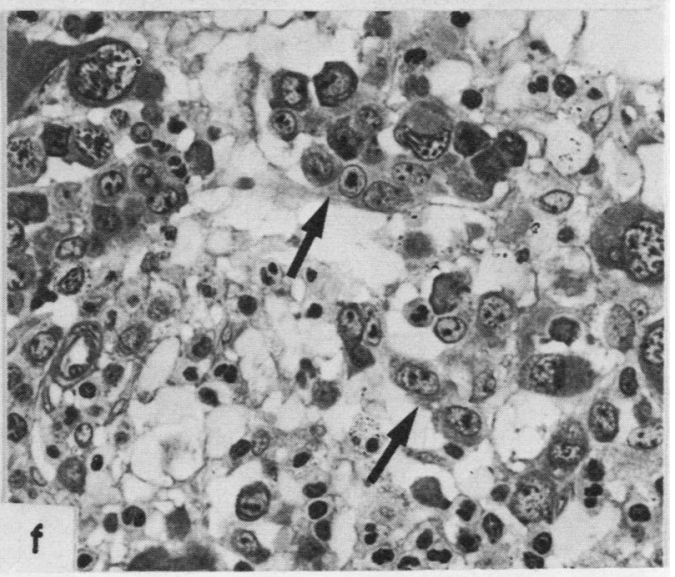

Fig. 1 Types of megakaryocytes as seen in bone marrow sections. (a) normal (b) dwarf (c) giant and pyknotic megakaryocytes (arrow) (d) immature (e) pleomorphic, with large round nuclei $(f)$ megakaryoblasts and immature megakaryocytes (arrows) (Gallamin blue Giemsa.) ( $\times 200 a-e ; \times 250$ b,f). 


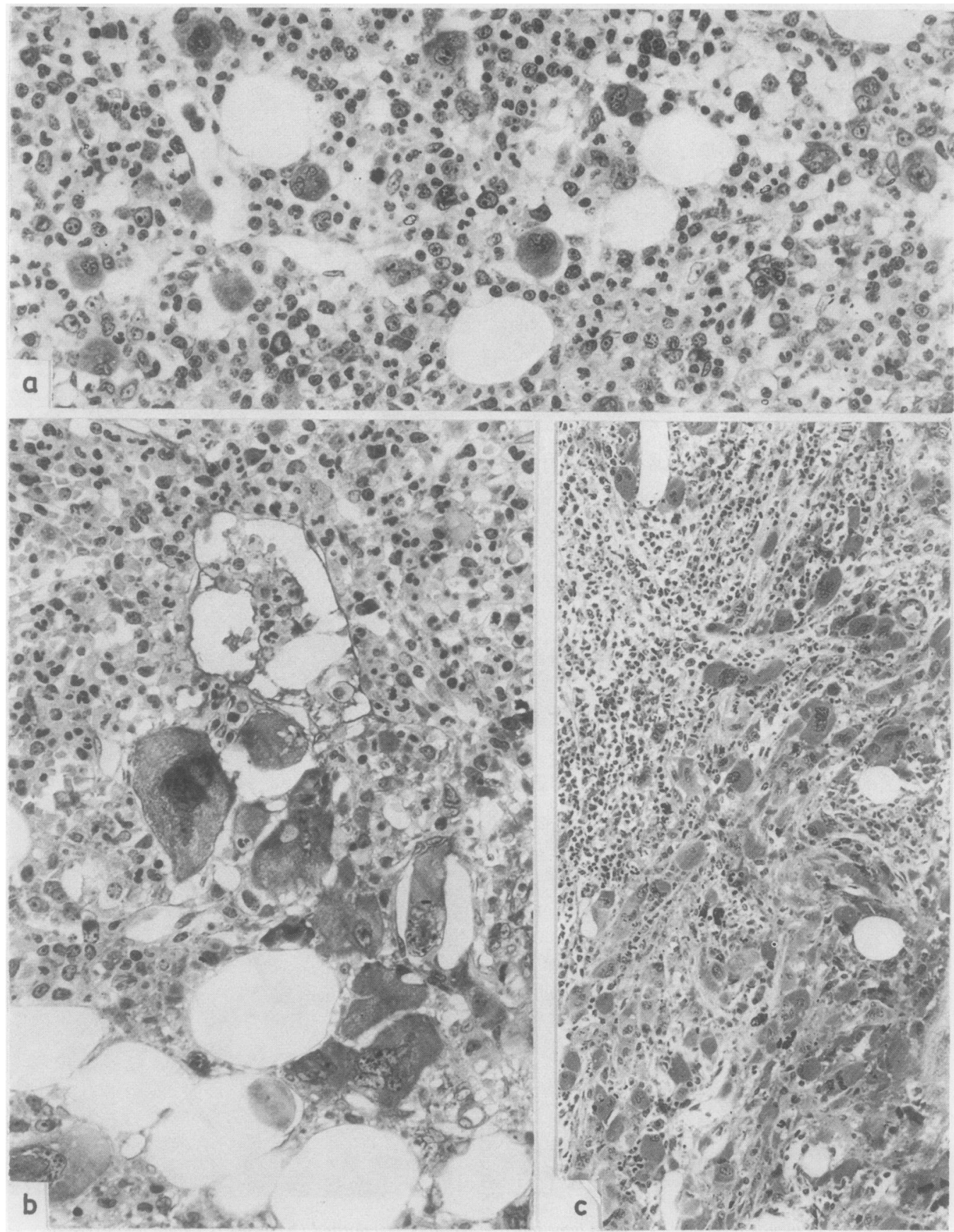

Fig. 2 Distribution of megakaryocytes in bone marrow. (a) mature and immature megakaryocytes of normal size showing diffuse distribution. (b) Cluster of giant sized megakaryocytes. (c) Sheet of mostly immature megakaryocytes. (Gallamin blue Giemsa.) (a) and (b) $\times 200 ; c \times 100$. 

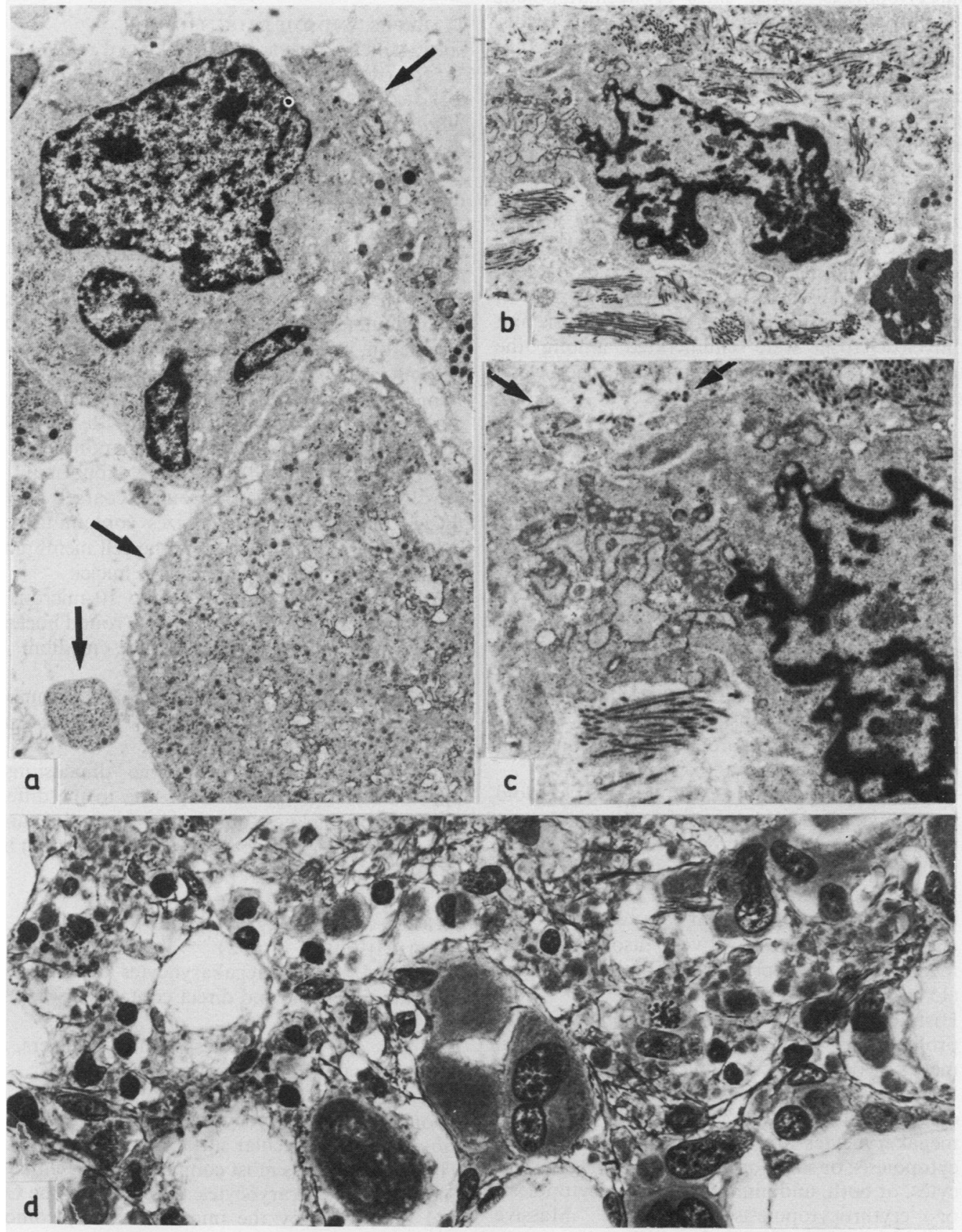

Fig. 3 Platelet production. (a) Megakaryocyte with cytoplasmatic demarcation and production of platelets (arrows) inside sinusoid; (b) Megakaryocyte in interstitium with platelets, surrounded by bundles of fibrils; (c) Detail of $(b)$ : part of megakaryocytic cytoplasm. Note proximity of fibrils to cytoplasmic surfaces (arrows); (d) Megakaryocytes and platelets in interstitial space, with incipient fibrosis. a-celectron micrographs; a $\times 1000$; $b \times 6300 ;$ and $c \times 12500 ; d \times 400$. Gomori's silver impregnation. 
abnormal megakaryocytes and immature granulocytopoiesis. Often islets of haematopoietic precursors within sinusoids with sclerotic walls. Clinical data: uncharacteristic, or syndrome of agnogenic myeloid metaplasia. Cases showing increase in reticular fibres or minor grades of myelofibrosis and osteomyelosclerosis were enumerated among the original groups, if these were identified. They were, however, separately evaluated.

5 Osteomyelosclerosis (160 cases) Bone biopsy and clinical data: as for myelofibrosis. In addition, irregular appositional bone formation and often an increase in fatty tissue. Cases showing increase in reticular fibres or minor grades of myelofibrosis and osteomyelosclerosis were enumerated among the original groups, if these were identified. They were, however, separately evaluated.

6 Blast crisis (38 cases) Bone biopsy data: presence of immature haematopoietic precursors with or without moderate reticular sclerosis, myelofibrosis, or osteomyelosclerosis. Clinical data: uncharacteristic or leukaemic blood picture and history of chronic myeloproliferative disorders.

7 Chronic myeloproliferative disorders not fitting into typical clinical groups

(i) Histology corresponds to polycythaemia vera. Clinical data consistent with chronic myeloproliferative disorders, but inconsistent with polycythaemia vera; (6 cases).

(ii) Histology corresponds to chronic myeloid leukaemia. Clinical data consistent with chronic myeloproliferative disorders, but inconsistent with chronic myeloid leukaemia; ( 7 cases).

(iii) Histology corresponds to primary thrombocythaemia. Clinical data consistent with chronic myeloproliferative disorders, but inconsistent with primary thrombocythaemia; (15 cases).

(iv) Histology corresponds to chronic myeloproliferative disorders, primarily abnormal megakaryocytopoiesis; clinical data uncharacteristic; (184 cases). Bone biopsy: high cellular density consisting either of giant, hyperploid megakaryocytes and partially mature granulocytopoiesis; or small or pleomorphic megakaryocytes, or both, and immature granulocytopoiesis or erythrocytopoiesis, or both. Massive reduction of fatty tissue, often with an increase in reticular fibres or incipient myelofibrosis or osteomyelosclerosis. Clinically, syndrome of agnogenic myeloid metaplasia, or refractory cytopenia(s).

\section{Results}

1 TYPES AND DISTRIBUTION OF MEGAKAR YOCYTES IN HISTOLOGICAL SUBGROUPS OF CHRONIC MYELOPROLIFERATIVE DISORDERS (FIG. 1)

Normal (Meg-N) Twenty-five to 50 microns in diameter, trilobulated to multilobulated nuclei with fine nuclear protein and rounded or polymorphous margins, usually with clear cytoplasmic demarcation. Dwarf (Meg-D) Fifteen to 25 microns in diameter, at least trilobulated nuclei, with fairly rounded margins.

Giant (Meg-G) Fifty to 120 microns in diameter, multilobulated nuclei with variable nuclear chromatin, irregular margins, often elongated with or without clear cytoplasmic demarcation.

Immature (Meg-I) Fifteen to 25 microns in diameter, round to ovoid nuclei with fine nuclear chromatin, and rounded or ovoid margins.

Pleomorphic (Meg-P) Various abnormal cells, mostly small with large round coarsely staining nuclei, or cells of various sizes containing several round separate nuclei close to the cell membrane, or multinucleated cells with blast-like nuclei.

Blast (Meg-B) Round cells 8 to 10 microns with deeply staining cytoplasm and large round nuclei; fine nuclear chromatin, one to three medium sized band-like nucleoli.

Pyknotic (Meg-Py) Either isolated clumps of nuclear material or bizarre nuclear masses surrounded by cellular debris.

It should be noted that the dimensions are approximations, and the error due to the difference between the thickness of sections ( 3 microns) and the diameter of megakaryocytes (15-120 microns) has to be taken into account. ${ }^{8}$

THREE FORMS OF DISTRIBUTION WERE DISTINGUISHED (FIG. 2)

Diffuse (Meg-d) Megakaryocytes diffusely spread near the sinusoids, and direct contact between single cells only.

Clusters (Meg-c) At least three clusters, each consisting of more than five megakaryocytes.

Sheets (Meg-s) At least one large or two small colonies of megakaryocytes, forming a layer or sheet mostly at the trabecular surface.

The Meg-d form is most commonly represented by $\mathbf{N}$ and $D$ type megakaryocytes, the $\mathrm{c}$ form by the $\mathrm{G}$ type, and the sform by the immature and pleomorphic types.

INTERSTITIAL DEPOSITION OF PLATELETS IN THE BONE MARROW (FIG. 3)

Normally, megakaryocytes shed their platelets into 
Table 3 Type and distribution of megakaryocytes in erythrocytic myelosis (polycythaemia vera)

\begin{tabular}{|c|c|c|c|c|c|c|c|}
\hline \multirow[t]{2}{*}{ Subgroups } & \multirow{2}{*}{$\begin{array}{l}\text { No of } \\
\text { patients }\end{array}$} & \multicolumn{3}{|c|}{ Megakaryocytic cell types } & \multicolumn{3}{|c|}{ Megakaryocytic proliferation forms } \\
\hline & & $N$ & $D$ & $G$ & $d$ & $c$ & $s$ \\
\hline $\begin{array}{l}\text { Erythrocytic proliferation } \\
\text { Erythrocytic/megakaryocytic/ } \\
\text { granulocytic proliferation }\end{array}$ & $\begin{array}{l}13 \\
95\end{array}$ & $\begin{array}{l}+t+ \\
+\end{array}$ & $\begin{array}{l}(+) \\
(+)\end{array}$ & $\begin{array}{l}(+) \\
++\end{array}$ & $\begin{array}{l}+++ \\
++\end{array}$ & $\overline{+}$ & - \\
\hline $\begin{array}{l}\text { Erythrocytic/megakaryocytic } \\
\text { proliferation }\end{array}$ & 62 & $(+)$ & - & +++ & $(+)$ & $+t+$ & - \\
\hline $\begin{array}{l}\text { Erythrocytic/granulocytic } \\
\text { proliferation }\end{array}$ & 17 & + & + & + & ++ & + & - \\
\hline
\end{tabular}

Table 4 Type and distribution of megakaryocytes in subgroups of granulocytic myelosis (chronic myeloid leukaemia)

\begin{tabular}{|c|c|c|c|c|c|c|c|}
\hline \multirow{2}{*}{$\begin{array}{l}\text { Subgroups (according to } \\
\text { predominant cell line) }\end{array}$} & \multirow{2}{*}{$\begin{array}{l}\text { No of } \\
\text { patients }\end{array}$} & \multicolumn{3}{|c|}{ Megakaryocytic cell types } & \multicolumn{3}{|c|}{ Megakaryocytic proliferation forms } \\
\hline & & $\bar{N}$ & $D$ & $\boldsymbol{G}$ & $d$ & $c$ & $\boldsymbol{s}$ \\
\hline $\begin{array}{l}\text { Granulocytic proliferation } \\
\text { Granulocytic/megakaryocytic } \\
\text { proliferation }\end{array}$ & $\begin{array}{r}25 \\
108\end{array}$ & $\stackrel{+}{(+)}$ & $\begin{array}{l}++ \\
+++\end{array}$ & - & $\begin{array}{l}++t \\
++\end{array}$ & $\bar{t}$ & - \\
\hline Pro-Granulocytic proliferation & 16 & $(+)$ & $+t+$ & - & ++ & + & - \\
\hline
\end{tabular}

Table 5 Type and distribution of megakaryocytes in subgroups of megakaryocytic myelosis, mature type (primary thrombocythaemia (Figs. 4a,b)

\begin{tabular}{|c|c|c|c|c|c|c|c|}
\hline \multirow{2}{*}{$\begin{array}{l}\text { Subgroups (according to } \\
\text { predominant cell line) }\end{array}$} & & \multicolumn{3}{|c|}{ Megakaryocytic cell types } & \multicolumn{3}{|c|}{ Megakaryocytic proliferation forms } \\
\hline & & $\bar{N}$ & $D$ & $G$ & $\bar{d}$ & $c$ & $s$ \\
\hline $\begin{array}{l}\text { Megakaryocytic proliferation-Gd } \\
\text { Megakaryocytic proliferation-Gc }\end{array}$ & $\begin{array}{l}\text { (14 cases) } \\
\text { ( } 44 \text { cases) }\end{array}$ & $\stackrel{+}{(+)}$ & - & $\begin{array}{l}+t \\
t+t\end{array}$ & $\begin{array}{l}+++ \\
(+)\end{array}$ & $\bar{t}++$ & - \\
\hline
\end{tabular}

Clinically typical primary thrombocythaemia was most often correlated with the megakaryocytic proliferation-Gd subgroup. The megakaryocytic proliferation-Gc subgroup probably represents a more advanced stage of megakaryocytic proliferation. Three cases of clinically typical primary thrombocythaemia with an immature megakaryocytic type (Meg-M-I) were seen.

Table 6 Type and distribution of megakaryocytes in subgroups of megakaryocytic-granulocytic myelosis, (Meg/Gran-M often subleukaemia (Figs. $4 c, d)$ )

\begin{tabular}{|c|c|c|c|c|c|c|c|}
\hline \multirow{2}{*}{$\begin{array}{l}\text { Subgroups (according to } \\
\text { predominant cell line) }\end{array}$} & & \multicolumn{3}{|c|}{ Megakaryocytic cell types } & \multicolumn{3}{|c|}{ Megakaryocytic proliferation forms } \\
\hline & & $\bar{N}$ & $D$ & $G$ & $d$ & $c$ & $s$ \\
\hline $\begin{array}{l}\text { Megakaryocytic proliferation-Gd } \\
\text { Megakaryocytic proliferation-Gc }\end{array}$ & $\begin{array}{l}\text { (61 cases) } \\
(67 \text { cases })\end{array}$ & $\begin{array}{l}(+) \\
(+)\end{array}$ & $(+)$ & $\begin{array}{l}+t \\
t+t\end{array}$ & $\begin{array}{l}+++ \\
(+)\end{array}$ & $\bar{t}++$ & - \\
\hline
\end{tabular}

The subgroup with diffusely distributed giant megakaryocytes comes closer to chronic myeloid leukaemia, the cluster subgroup to primary thrombocythaemia.

Table 7 Type and distribution of megakaryocytes in subgroups of immature, pleomorphic/megakaryocytic myelosis,Meg-M I/P (Fig. 5)

\begin{tabular}{|c|c|c|c|c|c|c|c|c|}
\hline \multirow{2}{*}{$\begin{array}{l}\text { Subgroups (according to } \\
\text { predominant cell line) }\end{array}$} & & \multicolumn{3}{|c|}{ Megakaryocytic cell types } & \multicolumn{3}{|c|}{ Megakaryocytic proliferation forms } & \multirow[b]{2}{*}{$s$} \\
\hline & & $N$ & $D$ & $G$ & $P / I$ & $d$ & $c$ & \\
\hline $\begin{array}{l}\text { Megakaryocytic proliferation-Pc } \\
\text { Megakaryocytic proliferation-Ps } \\
\text { Megakaryocytic proliferation-Is }\end{array}$ & $\begin{array}{l}\text { ( } 8 \text { cases) } \\
\text { (37 cases) } \\
(11 \text { cases) }\end{array}$ & $\begin{array}{l}(+) \\
(+)\end{array}$ & $\begin{array}{l}(+) \\
(+) \\
(+)\end{array}$ & $\begin{array}{l}(+) \\
(+) \\
-\end{array}$ & $\begin{array}{l}++ \\
+++ \\
++t\end{array}$ & $\begin{array}{l}- \\
+\end{array}$ & $\begin{array}{l}+++ \\
+ \\
-\end{array}$ & $\begin{array}{l}- \\
++ \\
++\end{array}$ \\
\hline
\end{tabular}

This group was separated from agnogenic myeloid metaplasia by its histology (Fig. 5); Fig. 5 also shows incipient myelofibrosis, a transformation especially common in this type.

the lumen of marrow sinusoids. In chronic myeloproliferative disorders megakaryocytes also release platelets into the interstitial spaces, and these deposits are often interwoven by a network of collagen fibres. Interstitial deposition of platelets is found when the megakaryocytes are clustered. 
Table 8 Mean (SD) blood cell counts of histological subgroups at diagnosis

\begin{tabular}{|c|c|c|c|}
\hline $\begin{array}{l}\text { Histological } \\
\text { subgroups }\end{array}$ & $\begin{array}{l}\text { Erythrocytes } \\
\times 10^{12} / 1\end{array}$ & $\begin{array}{l}\text { Leucocytes } \\
\times 10^{9} / 1\end{array}$ & $\begin{array}{l}\text { Thrombocytes } \\
\times 10^{9} / 1\end{array}$ \\
\hline $\begin{array}{l}\text { Erythrocytic proliferation-M } \\
2 \text { Granulocytic proliferation-M } \\
3 \text { Megakaryocytic proliferation-G-M } \\
\text { 4a Megakaryocytic proliferation-Gd- } \\
\text { granulocytic proliferation-M }\end{array}$ & $\begin{array}{l}7 \cdot 1(0 \cdot 9) \\
3 \cdot 8(0 \cdot 9) \\
4 \cdot 4(0 \cdot 8) \\
4 \cdot 3(1 \cdot 2)\end{array}$ & $\begin{array}{r}13 \cdot 7(6 \cdot 4) \\
111 \cdot 1(91 \cdot 5) \\
13 \cdot 7(14 \cdot 2) \\
23 \cdot 0(32 \cdot 7)\end{array}$ & $\begin{array}{l}372(211) \\
365(308) \\
865(380) \\
838(652)\end{array}$ \\
\hline $\begin{array}{l}\text { 4b Megakaryocytic proliferation-Gc- } \\
\text { granulocytic proliferation-M }\end{array}$ & $4 \cdot 3(1 \cdot 1)$ & $22 \cdot 0(16 \cdot 7)$ & $498(548)$ \\
\hline $\begin{array}{l}5 \text { Megakaryocytic immature/pleomorphic } \\
\text { proliferation myelosis-M }\end{array}$ & $3.1(1.2)$ & $18.9(37.4)$ & $359(648)$ \\
\hline $\begin{array}{l}\text { Myelofibrosis grade } 2 \\
\text { Osteomyelosclerosis grades } 2 \text { and } 3 \\
\text { Blast crisis }\end{array}$ & $\begin{array}{l}3 \cdot 8(1 \cdot 6) \\
3 \cdot 6(1 \cdot 1) \\
3 \cdot 1(1 \cdot 4)\end{array}$ & $\begin{array}{l}15 \cdot 1(20 \cdot 2) \\
12 \cdot 5(16 \cdot 4) \\
16 \cdot 4(26 \cdot 9)\end{array}$ & $\begin{array}{l}211(221) \\
205(162) \\
130(178)\end{array}$ \\
\hline
\end{tabular}

*The subgroups and blast crisis include grades 1 of myelofibrosis and osteomyelosclerosis. These fibrotic subgroups differ from their non-fibrotic equivalents by lower mean values of erythrocytes and thrombocytes and by higher mean values of leucocytes in subgroups 1,2 , and 5 .

\section{HISTOLOGICAL SUBGROUPS AND}

MEGAKARYOCYTIC ANOMALIES (TABLES 3-7)

Pyknotic megakaryocytes increased as the megakaryocyte count rose, especially with clusters of giant cells. Pleomorphic types were rare (Table 4). In chronic myeloid leukaemia megakaryocytes were either normal or often of the dwarf type. Combinations of granulocytic and giant megakaryocytic proliferations formed a separate group (Table 6). Eosinophilic variants of chronic myeloid leukaemia occurred in all subgroups, most commonly in the granulocytic proliferation megakaryocytic proliferation subgroup; basophilic variants were extremely rare (Tables 5-7).

\section{GRADES OF MYELOFIBROSIS AND} OSTEOMYELOSCLEROSIS; BLAST CRISIS (FIG. 6) Myelofibrosis For grade 1 at least three coarse fibre bundles were found within a microscopic field at a magnification of $\times 200$. For grade 2 there was a reduction of haematopoiesis accompanied by fibrosis around blood vessels, trabecular bone, or cells. In advanced cases the residual haemapoiesis consisted mainly of clusters of giant, immature, or pleomorphic megakaryocytes, erythroblasts or promyelocytes.

Osteomyelosclerosis For grade 1 there was at least one area per section with appositional (occasionally interstitial) new bone formation. For grade 2 there were more than three areas per section of immature

Table 9 Correlation of histological subgroups with blood cell counts at diagnosis (percentages of each histological subgroup belonging to haematological categories)

\begin{tabular}{|c|c|c|c|c|c|c|c|c|}
\hline $\begin{array}{l}E \\
L \\
T\end{array}$ & $\begin{array}{l}A 1 \\
>6 \cdot 5 \\
<30 \\
348 \\
\text { (mean) } \\
\text { (153 cases) }\end{array}$ & $\begin{array}{l}A 2 \\
5 \cdot 5-6 \cdot 5 \\
<30 \\
422 \\
\text { (mean) } \\
\text { (71 cases) }\end{array}$ & $\begin{array}{l}B 1 \\
<5 \cdot 5 \\
>30 \\
349 \\
\text { (mean) } \\
\text { (151 cases) }\end{array}$ & $\begin{array}{l}B 2 \\
<5 \cdot 5 \\
15-30 \\
492 \\
\text { (mean) } \\
\text { (119 cases) }\end{array}$ & $\begin{array}{l}C 1 \\
<5.5 \\
<15 \\
>700 \\
\text { (48 cases) }\end{array}$ & $\begin{array}{l}C 2 \\
<5.5 \\
<15 \\
300-700 \\
\text { (69 cases) }\end{array}$ & $\begin{array}{l}D \\
<5.5 \\
<15 \\
<300 \\
\text { (219 cases) }\end{array}$ & $\begin{array}{l}M \\
>5 \cdot 5 \\
>30 \\
446 \\
\text { (mean) } \\
(20 \text { cases }\end{array}$ \\
\hline $\begin{array}{l}1 \text { Erythrocytic proliferation-M } \\
\text { (200 cases) }\end{array}$ & $72^{*}$ & $25^{*}$ & - & 0.5 & 0.5 & - & - & 2 \\
\hline $\begin{array}{l}2 \text { Granulocytic proliferation- } M \\
\text { (132 cases) }\end{array}$ & - & - & $82^{*}$ & 14 & - & - & 2 & 2 \\
\hline $\begin{array}{l}3 \text { Megakaryocytic proliferation-G-M } \\
\text { (58 cases) }\end{array}$ & - & 4 & 2 & 19 & $47^{*}$ & $28^{*}$ & - & 0 \\
\hline $\begin{array}{l}\text { 4a Megakaryocytic proliferation-Gd- } \\
\text { granulocytic proliferation-M } \\
\text { (61 cases) }\end{array}$ & - & 7 & 18 & $48 *$ & 8 & 8 & 8 & 3 \\
\hline $\begin{array}{l}\text { 4b Megakaryocytic proliferation-Gc- } \\
\text { granulocytic proliferation-M }\end{array}$ & - & 9 & 4 & $24^{*}$ & 16 & $24^{*}$ & 15 & 8 \\
\hline $\begin{array}{l}(67 \text { cases) } \\
5 \text { Megakaryocytic immature/pleomorphic } \\
\text { proliferation-M (56 cases) }\end{array}$ & 4 & - & 11 & 18 & 5 & 5 & $57^{*}$ & - \\
\hline $\begin{array}{l}\text { Grade } 2 \text { myelofibrosis } \\
\text { ( } 78 \text { cases) }\end{array}$ & 4 & 6 & 6 & 14 & - & 6 & $61^{*}$ & 3 \\
\hline $\begin{array}{l}\text { Grade } 2 \text { and } 3 \text { osteomyelosclerosis } \\
\text { Blast crisis } \\
\text { (38 cases) }\end{array}$ & $\begin{array}{l}1 \\
5\end{array}$ & $\underline{2}$ & $\begin{array}{r}7 \\
16\end{array}$ & $\begin{array}{l}12 \\
11\end{array}$ & $\begin{array}{l}1 \\
3\end{array}$ & $\begin{array}{r}14 \\
3\end{array}$ & $\begin{array}{l}62 * \\
62 *\end{array}$ & 1 \\
\hline \multicolumn{9}{|c|}{$\begin{array}{l}\text { Horizontal: histological subgroups; (according to } 1-5 \text { plus myelofibrosis, osteomyelosclerosis, and blast crisis). Vertical: haematological categories; } \\
\mathrm{E}=\text { erythrocytes, } \mathrm{L}=\text { leucocytes, } \mathrm{T}=\text { thrombocytes. } \\
\text { *Significant values. }\end{array}$} \\
\hline
\end{tabular}


bone or moderate thickening of the trabeculae, or both. In grade 3 osteosclerosis with severely abnormal trabecular architecture was seen with small marrow spaces and often increased osseous remodelling. Marrow changes were similar to those of grade 2 myelofibrosis or were of an oedematous and fatty marrow.

Blast crisis Blast crisis may consist of one or more cell lines, especially megakaryoblasts and promyelocytes or proerythroblasts. The blasts may be arranged in sheets in paratrabecular or perivascular areas, or occupy the whole intertrabecular spaces as in the "packed marrow" of acute leukaemia. Blast crisis was found without myelofibrosis or osteomyelosclerosis in 12 cases, and combined with myelofibrosis or osteomyelosclerosis in 26 cases.

\section{CORRELATIONS OF HISTOLOGICAL SUBGROUPS WITH BLOOD CELL COUNTS AT DIAGNOSIS}

Table 8 shows the mean blood cell values of the major subgroups. This shows that the histological manifestations of untreated cases generally correspond to characteristic blood cell counts. Table 9 gives a more differentiated test of the haematological validity of the histological subgroups. There was a good correlation between the histological erythrocytic proliferation and granulocytic proliferation groups and their haematological expression, typical of polycythaemia vera and chronic myeloid leukaemia,

Table 10

\section{Correlations of clinical data for age and sex:}

Age older in:

Myelofibrosis than Osteomyelosclerosis

Megakaryocytic proliferation-Gd-Granulocytic proliferation-M than Granulocytic proliferation-M

Chronic myeloproliferation disorders $/ B_{2}$ than $B_{1}$

Chronic myeloproliferation disorders/Megakaryocytic cell type $G$ rather than Megakaryocytic cell type $D * *$

Megakaryocytic proliferation-Gc-Granulocytic proliferation-M than Megakaryocytic proliferation-Gd-Granulocytic proliferation-M

$$
\begin{aligned}
\} p & =0.0001 \\
p & =0.001 \\
p & =0.01
\end{aligned}
$$

Higher prevalence among women in:

Chronic myeloproliferation disorders/Megakaryocytic proliferation form $\mathrm{c}$ than Megakaryocytic proliferation form $\mathrm{d}^{* *}$

Chronic myeloproliferation disorders/Megakaryocytic cell type $G$ rather than other Megakaryocytic cell types**

Chronic myeloproliferation disorders $T>700$ tsd than $T<700$ tsd

Chronic myeloproliferation disorders/L $<30$ tsd than $\mathrm{L}>30$ tsd*

Primary thrombocythalomin than other Chronic myeloproliferation disorders

Chronic myeloproliferation disorders $/ C_{1}$ than $B_{1}$

Chronic myeloproliferation disorders $/ C_{2}$ than $B_{2} *$

Megakaryocytic proliferation-Gd-Granulocytic proliferation-M than Granulocytic proliferation-M

Megakaryocytic proliferation-Gc-Granulocytic proliferation-M than Granulocytic proliferation-M

$$
\begin{aligned}
& p=0.0001 \\
& p=0.0001 \\
& p=0.001 \\
& p=0.001 \\
& p=0.001 \\
& p=0.001 \\
& p=0.007 \\
& p=0.03 \\
& p=0.04
\end{aligned}
$$

\section{Correlations of histological data:}

Blast crisis more common in:

Myelofibrosis/Osteomyelosclerosis than other chronic myeloproliferative disorders Myelofibrosis than Osteomyelosclerosis

Interstitial deposition of platelets more common in:

Myelofibrosis/Osteomyelosclerosis than other chronic myeloproliferative disorders

Chronic myeloproliferative disorders Megakaryocytic proliferation form $G^{* *}$ than other forms

$$
\begin{aligned}
& p=0.0001 \\
& p=0.005 \\
& p=0.001 \\
& p=0.001
\end{aligned}
$$

\section{Correlations of megakaryocytic anomalies:}

Diffuse distribution/Megakaryocytic proliferations more common in. Chronic myeloproliferative disorders $/ \mathrm{L}>30$ tsd than $\mathrm{L}<30$ tsd Chronic myeloproliferative disorders $/ T<700$ tsd than $T>700$ tsd

Cluster distribution/Megakaryocytic proliferations more common in: Myelofibrosis/Osteomyelosclerosis than other Chronic myeloproliferative disorderst Chronic myeloproliferative disorders/ $\mathrm{L}<30$ tsd than $\mathrm{L}>30$ tsd Chronic myeloproliferative disorders $/ \mathrm{T}>700$ tsd than $\mathrm{T}<700$ tsd

Sheet distribution/Megakaryocytic proliferations more common in: Blast crisis than other Chronic myeloproliferative disorderst Myelofibrosis/Osteomyelosclerosis than other Chronic myeloproliferative disorders $†$

Pleomorphic and immature Megakaryocytic cell types more common in: Blast crisis than other Chronic myeloproliferative disorderst Myelofibrosis/Osteomyelosclerosis than other Chronic myeloproliferative disorders $\dagger$

Pyknotic Megakaryocytes more common in:

Myelofibrosis/Osteomyelosclerosis than other Chronic myeloproliferative disorders

*all cases of Chronic myeloproliferative disorders

* Chronic myeloproliferative disorders with increase in megakaryocytes only

texcept of Blast crisis

fexcept of Myelofibrosis/Osteomyelosclerosis

$\mathrm{T}=$ thrombocytes; $\mathrm{L}=$ leucocytes 

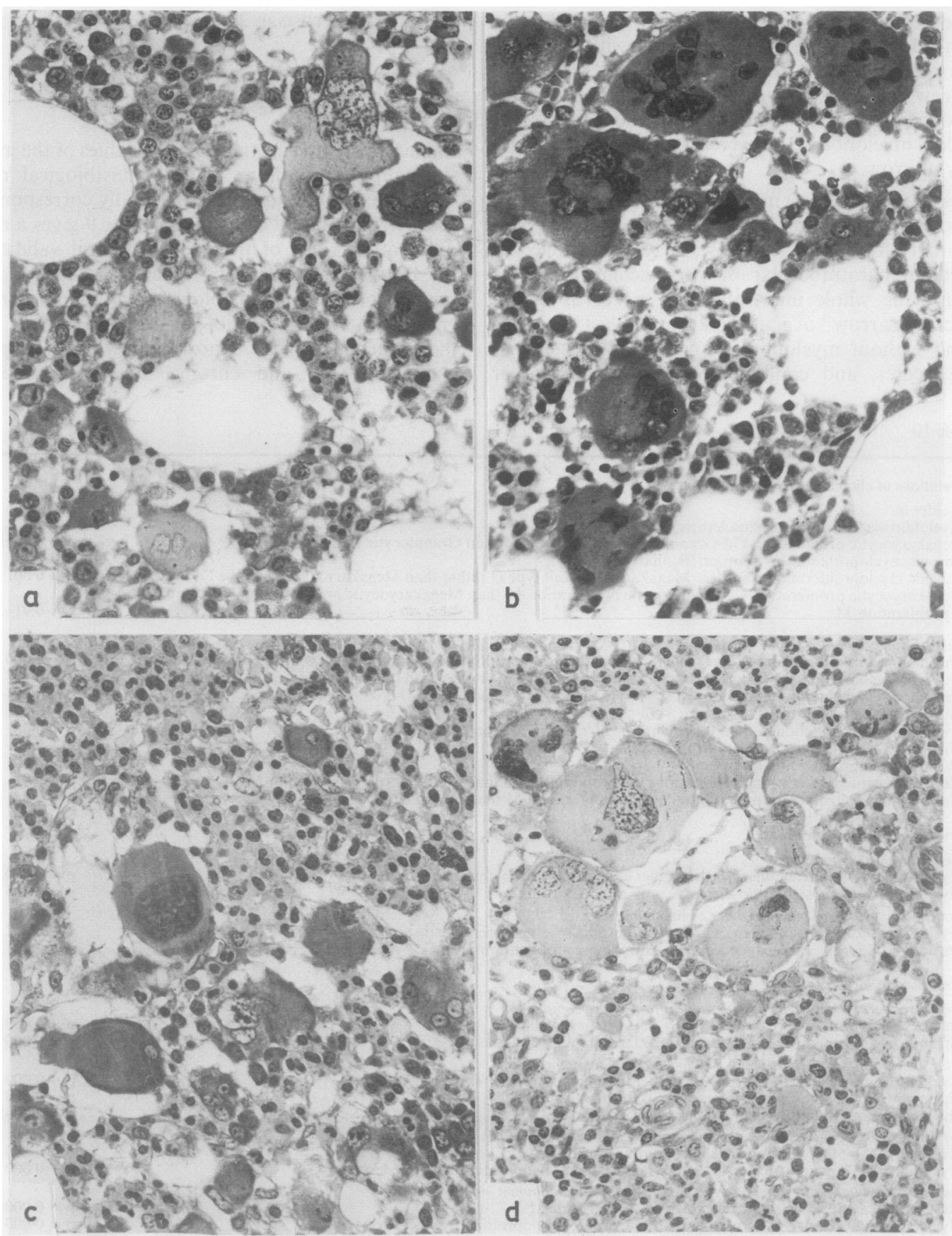

Fig. 4 Megakaryocytic proliferation $M$ mature ( $d G$ or $c G$ ) = primary thrombocythaemia; two subgroups.

(a) Unilinear megakaryocytic dG, (woman 68 years): red blood cells $4.5 \times 10^{12} / l$, white cells $11 \times 10^{9} / l$, platelets $2.800 \times 10^{9} / l$. (b) Unilinear megakaryocytic $c G$ (man 37 years): red blood cells $4.8 \times 10^{12} / l$, white cells $10 \times 10^{9} / l$, platelets $1300 \times 10^{9} / l$.

Megakaryocytic proliferation-granulocytic proliferation-M ( $d G$ and $c G)$; two subgroups. (c) Bilinear megakaryocytic granulocytic proliferation-dG, (man 76 years), red blood cells $4.7 \times 10^{12} / l$, white cells $16 \times 10^{9} / l$, platelets $271 \times 10^{9} / l$. (d) Bilinear Megakaryocytic-granulocytic proliferation-cG; (woman 67 years): red blood cells $3.4 \times 10^{12} / l$, white cells $13 \times 10^{9} / l$, platelets $186 \times 10^{9} / \mathrm{l}$; (Gallamin blue and Giemsa.) $a, c, d \times 200 ; b$ $\times 250$. 

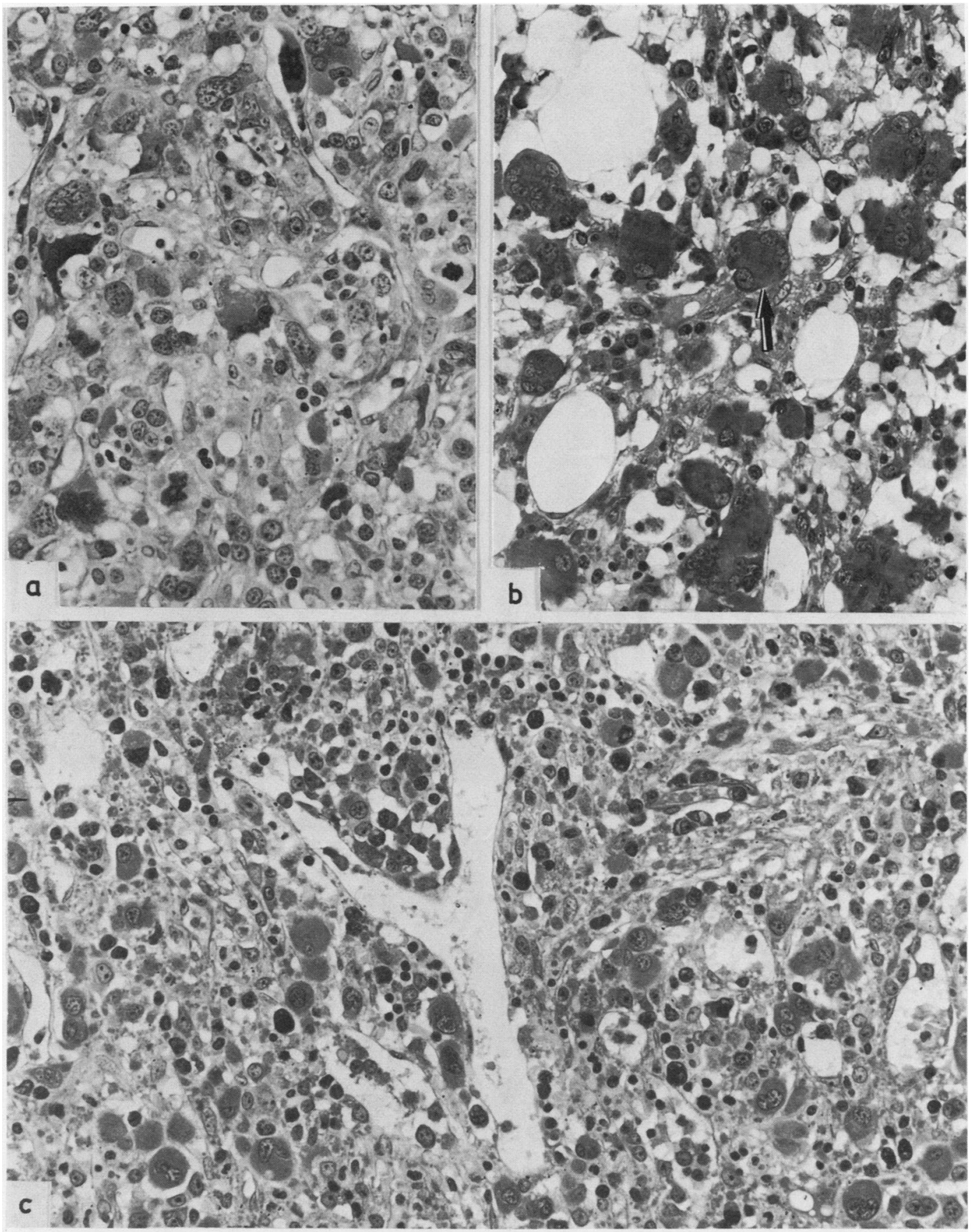

Fig. 5 Megakaryocytic immature and pleomorphic proliferation M; two subgroups. (a) Megakaryocytic proliferation-M-P; predominantly pleomorphic megakaryocytes showing large nuclei with incomplete lobulation (woman 64 years): red blood cells $2.6 \times 10^{12} / l$, white cells $2 \times 10^{9} / l$, platelets $12 \times 10^{9} / l \times 200$. (b) Megakaryocytic proliferation M-P; among greatly increased megakaryocytes the type with ring shaped arrangement of multiple single nuclei prevails (arrow) (woman 65 years): red blood cells $3.3 \times 10^{12} / l$, white cells $8 \times 10^{9} / l$, platelets $28 \times 10^{9} / l$. (c) Megakaryocytic proliferation M-I; masses of immature and pyknotic megakaryocytes are prevalent, sometimes with interstitial deposition of platelets and myelofibrosis (man 63 years): red blood cells $2.4 \times 10^{12} / l$, white cells $35 \times 10^{9} / l$, platelets $1000 \times 10^{9} / l$. (Gallamin blue and Giemsa). 

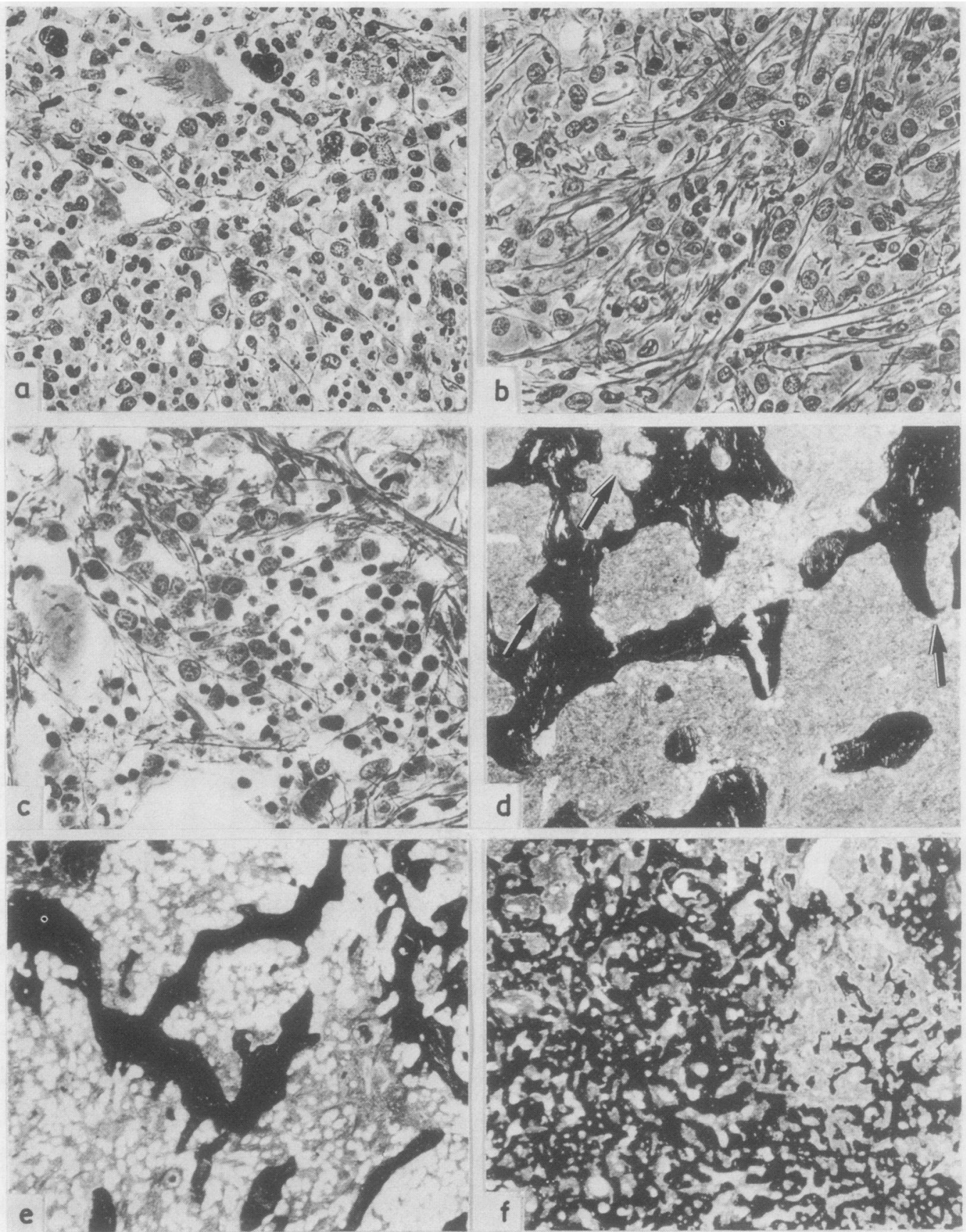

Fig. 6 Myelofibrosis and osteomyelosclerosis. (a) Reticular sclerosis; (b) myelofibrosis grade 1; (c) myelofibrosis grade 2. (Gomori's silver impregnation) magnification $a \times 200 ; b$ and $c \times 250$. (d) osteomyelosclerosis grade 1; spicules of primitive bone (arrows); (e) osteomyelosclerosis grade 2: irregular osteocondensation; (f) osteomyelosclerosis grade 3: filigree meshwork of osteoid, having only small marrow spaces. (Gomori's silver impregnation.) $\times 10$. 
respectively. It should be noted, however, that erythrocytosis and leukaemia also occur in a small proportion of all the other groups. Giant cell megakaryocytic myelosis and thrombocythaemia, the typical combination found in primary thrombocythaemia, are also clearly correlated, although thrombocythaemia $\left(>700 \times 10^{9} / 1\right)$ is found in the other groups also (for example in $50 \%$ of Meg-GdGran-M). The mixed megakaryocytic-granulocytic proliferation groups are clearly distinguished from each other. Pancytopenia was almost as common in the groups of megakaryocytic-I/P-M, myelofibrosis, osteomyelosclerosis, and blast crisis, although minor percentages of these groups exhibit erythraemia, leukaemia and thrombocythaemia. The incidence of Grade 1 myelofibrosis and osteomyelosclerosis were included in the corresponding histological subgroups in which the following percentages were found: erythrocytic proliferation-M $6 \%$ and $2 \%$, respectively; granulocytic proliferation-M $7 \%$ and $0 \%$, respectively; megakaryocytic proliferation-G-M $7 \%$ and $2 \%$, respectively; megakaryocytic proliferation-Gdgranulocytic proliferation-M $5 \%$ and $5 \%$, respectively; megakaryocytic proliferation-Gc-Granulocytic proliferation-M $35 \%$ and $4 \%$, respectively; megakaryocytic-I/P-M $45 \%$ and $7 \%$, respectively; blast crisis $51 \%$ and $16 \%$, respectively. These figures show that in all subgroups minor grades of myelofibrosis are much more common than osteomyelosclerosis, the highest percentages of myelofibrosis and osteomyelosclerosis occurring in the cluster variant of mixed megakaryo-cyticgranulocytic myelosis, and in the immature and pleomorphic megakaryocytic and blastic subgroups.

4 STATISTICAL EVALUATION OF CLINICAL AND HISTOLOGICAL CRITERIA

Correlations between clinical and histological data were statistically checked (Table 10 ). Especially noteworthy were: the mean age, which was higher in myelofibrosis than in osteomyelosclerosis; the fact that women predominate in the groups with thrombocythaemia; splenomegaly was more common in the megakaryocytic-granulocytic proliferation group in which there were clusters rather than diffusely distributed megakaryocytes, and was also commoner in myelofibrosis/osteomyelosclerosis than in the rest of chronic myeloproliferative disorders.

\section{SEQUENTIAL BIOPSIES (TABLE 11)}

Transitions between the different entities within the chronic myeloproliferative disorders were common and only $19 \%$ of the 169 sequential biopsies had the same histological picture as in the initial biopsies. Progression of disease was indicated by an increase of megakaryocytes and by myelofibrosis/osteomyelosclerosis. Follow up studies showed that fibrosis and blastic metamorphosis may occur at the same time.

\section{SURVIVAL ANALYSIS AND WORKING CLASSIFICATION}

When the survival of the haematological groups was compared with those of the histological groups the latter showed a broader range (Table 12) but their median survivals were similar, confirming that the haematological and histological criteria chosen for this study characterised the main clinical entities of chronic myeloproliferative disorders (polycythaemia vera, chronic myeloid leukaemia, primary thrombocythaemia), to which about a third of the patients studied belonged. Another third of patients with chronic myeloproliferative disorders were distinguished by a greater variety of histological changes, had less typical blood values, and were more difficult to classify. These were the atypical or intermediate cases, which often transformed into

Table 11 Sequential biopsies (295) of 126 patients with chronic myeloproliferative disorders who were untreated at initial biopsy

\begin{tabular}{|c|c|c|c|c|c|c|c|c|c|}
\hline \multirow{3}{*}{$\begin{array}{l}\text { No of } \\
\text { patients }\end{array}$} & \multirow{3}{*}{$\begin{array}{l}\text { No of } \\
\text { biopsies }\end{array}$} & \multicolumn{8}{|c|}{ Histological picture at: } \\
\hline & & \multirow[t]{2}{*}{ Initial biopsy } & \multicolumn{7}{|c|}{ Sequential biopsy } \\
\hline & & & $\begin{array}{l}\text { No } \\
\text { change }\end{array}$ & Gran-M & $\begin{array}{l}\text { Meg-Gc- } \\
\text { Gran-M }\end{array}$ & $M e g-I / P-M$ & $\boldsymbol{M F}$ & $O M S$ & $B C$ \\
\hline $\begin{array}{r}36 \\
16 \\
5 \\
6 \\
17 \\
16 \\
11 \\
19\end{array}$ & $\begin{array}{l}91 \\
38 \\
10 \\
14 \\
41 \\
38 \\
23 \\
40\end{array}$ & $\begin{array}{l}\text { Ery-M (PV) } \\
\text { Gran-M (CML) } \\
\text { Meg-G-M (PT) } \\
\text { Meg-Gd-Gran-M } \\
\text { Meg-Gc-Gran-M } \\
\text { Meg-I/P-M } \\
\text { MF } \\
\text { OMS }\end{array}$ & $\begin{array}{l}10 \\
1 \\
1 \\
1 \\
4 \\
+(\text { Fibr-) } \\
4 \\
2\end{array}$ & $\begin{array}{l}12 \\
1 \\
1(+M F)\end{array}$ & $\begin{array}{l}3 \\
2 \\
2 \\
2+ \\
1\end{array}$ & $\begin{array}{l}2 \\
3 \\
1 \\
2 \\
4+\end{array}$ & $\begin{array}{l}3 \\
4 \\
2 \\
1 \\
4 \\
5 \\
3+\end{array}$ & $\begin{array}{l}6 \\
5 \\
1 \\
3 \\
5 \\
1+ \\
17+\end{array}$ & $\begin{array}{l}1 \\
1 \\
2 \\
2\end{array}$ \\
\hline
\end{tabular}

Mean interval between first and last biopsy $=36$ months. †Increase of changes of same type.

Key to abbreviations:

PV = polycythaemia vera; $C M L=$ chronic myeloid leukaemia; $P T=$ primary thrombocythaemia; $\mathbf{M F}=$ myelofibrosis; OMS = osteomyelosclerosis; $\mathrm{BC}=$ blast crisis; Ery = erythrocytic proliferation; Gran = granulocytic proliferation; Meg = megakaryocytic proliferation 
Table 12 Median survival of patients with chronic myeloproliferative disorders grouped according to blood cell values

\begin{tabular}{|c|c|c|c|c|c|c|}
\hline \multirow[t]{2}{*}{ Group } & \multirow{2}{*}{$\begin{array}{l}\text { Erythrocytes } \\
\left(\times 10^{12} / l\right)\end{array}$} & \multirow{2}{*}{$\begin{array}{l}\text { Leucocytes } \\
\left(\times 10^{9} / l\right)\end{array}$} & \multirow{2}{*}{$\begin{array}{l}\text { Thrombocytes } \\
\left(\times 10^{9} / l\right)\end{array}$} & \multirow{2}{*}{$\begin{array}{l}\text { Survival } \\
\text { (median) } \\
\text { (months) }\end{array}$} & \multicolumn{2}{|l|}{ Histological groups } \\
\hline & & & & & Group & $\begin{array}{l}\text { Survival } \\
\text { (median) } \\
\text { (months) }\end{array}$ \\
\hline$\overline{\mathrm{Al}}$ & $>6.5$ & $<30$ & $\begin{array}{l}348 \\
\text { (mean) }\end{array}$ & 112 & $\begin{array}{l}1 \text { Erythrocytic proliferation-M } \\
2 \text { Erythrocytic proliferation-M borderline }\end{array}$ & $\begin{array}{l}130 \\
114\end{array}$ \\
\hline A2 & $5 \cdot 5-6 \cdot 5$ & $<30$ & $\begin{array}{l}422 \\
\text { (mean) }\end{array}$ & 101 & $\begin{array}{l}3 \text { Megakaryocytic proliferation-G-M } \\
4 \text { Granulocytic proliferation-M } \\
5 \text { Megakaryocytic proliferation-Gd +c- } \\
\text { granulocytic proliferation-M }\end{array}$ & $\begin{array}{r}114 \\
34 \\
31\end{array}$ \\
\hline B1 & $<5 \cdot 5$ & $>30$ & $\begin{array}{l}349 \\
\text { (mean) }\end{array}$ & 31 & $\begin{array}{l}6 \text { Myelofibrosis/osteomyelosclerosis } \\
7 \text { Megakaryocytic proliferation-I/P-M }\end{array}$ & $\begin{array}{l}30 \\
14\end{array}$ \\
\hline B2 & $<5 \cdot 5$ & $15-30$ & $\begin{array}{l}492 \\
\text { (mean) }\end{array}$ & 33 & $\begin{array}{l}8 \text { Blast crisis } \\
9 \text { Blast crisis/myelofibrosis }\end{array}$ & $\begin{array}{l}6 \\
4\end{array}$ \\
\hline $\begin{array}{l}\mathrm{Cl} \\
\mathrm{C} 2 \\
\mathrm{D} \\
\mathrm{M}\end{array}$ & $\begin{array}{l}<5.5 \\
<5.5 \\
<5.5 \\
>5.5\end{array}$ & $\begin{array}{l}<15 \\
<15 \\
<15 \\
>30\end{array}$ & $\begin{array}{l}700 \\
300-700 \\
300 \\
446 \\
\text { (mean) }\end{array}$ & $\begin{array}{r}124 \\
31 \\
22 \\
39\end{array}$ & & \\
\hline
\end{tabular}

Significant differences of survival

Erythrocytic proliferation-M : Granulocytic proliferation-M

Erythrocytic proliferation-M : Megakaryocytic proliferation-Gc-granulocytic proliferation-M

Erythrocytic proliferation-M : Megakaryocytic proliferation-Gd-granulocytic proliferation-M

Granulocytic proliferation-M : Megakaryocytic proliferation-G-M

myelofibrosis/osteomyelosclerosis or blast crisis. The remaining third of the cases defined according to their histology showing blastic proliferation, or myelofibrosis/osteomyelosclerosis, or a combination of both, were therefore considered to be the transformed stages of chronic myeloproliferative disorders. In each of these three groups different types and forms of distribution of megakaryocytic proliferation predominated (Table 13).

These results lead to the formulation of a working classification (Table 13) consisting of three main categories. The subgroups were distinguished by the proliferating cell lines, their maturity, the degree of fibrosis and osteosclerosis, the distribution and cytological types of megakaryocytes, and the degree of efficient platelet production. This working classification includes all structural forms found in chronic myeloproliferative disorders, together with their initial haematological and clinical correlations.

\section{Discussion}

Knowledge of how chronic myeloproliferative disorders is expressed in the bone marrow contributes to a better understanding of the myeloproliferative process and the prognosis of individual patients. ${ }^{3}$ Recent biopsy techniques, however, have disclosed several atypical cases (about one third of this series). These can be identified at initial diagnosis or in the later stages of the disease and thus may be integral parts of the natural history of the chronic myeloproliferative disorders. To test this our study had to consider all cases in which a disease of chronic myeloproliferative disorder was assumed. The clinical information available was used to distinguish typical from atypical cases, and simple haematological categories were arbitrarily chosen as counterparts to the histological evaluation. The major histological categories of this system coincided broadly with the classic clinical groups, thus providing a haematological framework in which to correlate the histological background of the cases and the clinical features.

Different types of normal megakaryocytes and megakaryocytic anomalies have long been recognised in all the groups of chronic myeloproliferative disorders, ${ }^{91011}$ but have not yet been classified. Myelofibrosis/osteomyelosclerosis originates from chronic myeloproliferative disorders and disturbances of platelet production are among their causative factors. ${ }^{913}$ Agnogenic myeloid metaplasia is the clinical correlate of myelofibrosis/ osteomyelosclerosis; primary thrombocythaemia has been recognised as the expression of a special mature form of megakaryocytic myelosis. ${ }^{313}$

Two histological processes exert a major influence on the natural history of chronic myeloproliferative disorders: the participation of the different myeloid cell lines; and the disintegration of megakaryocytes and platelets. ${ }^{12}$ Pure megakaryocytic proliferation with isolated thrombocythaemia is as rare as pure erythrocytosis. Both are compatible with bone marrow function and have a good prognosis, unlike the proliferation of granulocytic precursors in chronic myeloid leukaemia. The participation of granulocytic 


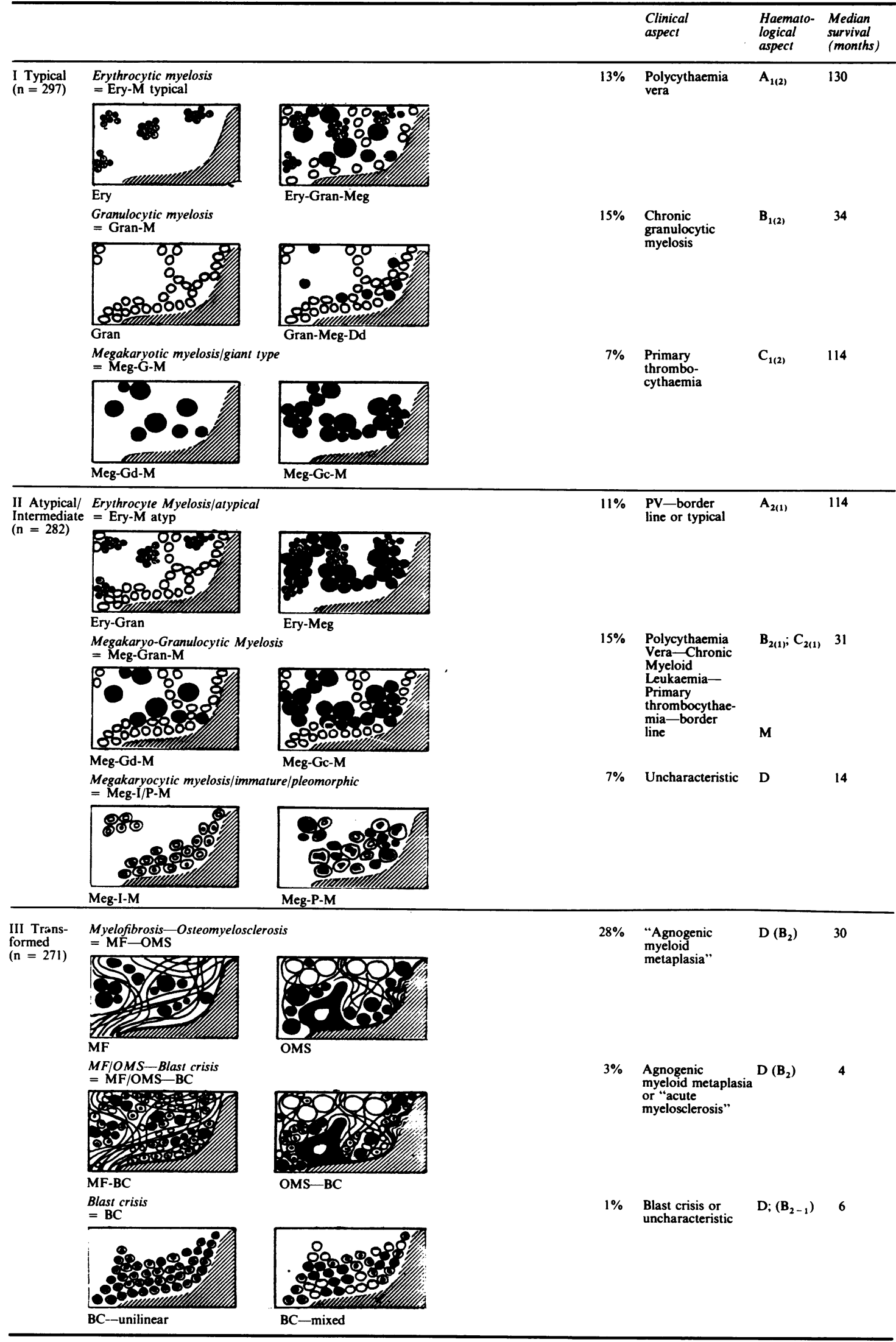


proliferation in neoplasias of the two other cell lines adversely influences the course of chronic myeloproliferative disorders, mainly towards blast crisis. ${ }^{14}$

Megakaryocytic pathology has a role of its own in chronic myeloproliferative disorders. The dwarf type is characteristic of granulocytic proliferation-M (chronic myeloid leukaemia), with a tendency to develop into immature forms. ${ }^{3}$ The occurrence of sheets is related to immature and pleomorphic megakaryocytes and thrombocytopenia. The incidence of the combination of fibrotic and blastic changes (equivalent to "acute myelosclerosis" in many cases) points to a closely related pathogenesis. The giant type of megakaryocyte occurs with increased megakaryocytopoiesis, especially in polycythaemia vera, primary thrombocythaemia, and megakaryocytic granulocytic proliferation- $M$. The diffuse form of the megakaryocytic granulocytic proliferation- $M$ is prone to subleukaemic changes and splenomegaly, whereas the cluster form tends towards thrombocythaemia and myelofibrosis, due in part to interstitial deposits of platelets and interstitial degeneration of megakaryocytes.

The results of this study lead to a closer correlation of the different histological and haematological expressions of chronic myeloproliferative disorders; to a better definition of the atypical forms; and to the integration of all entities into a proposed classification that indicates the place of each group in the sequence of the myeloproliferative processes and their transformations. With this working classification it will be possible to classify an individual patient with chronic myeloproliferative disorders more clearly and in doing so coordinate as much information as possible from all other sources.

\section{References}

${ }^{1}$ Dameshek W. Some speculations on the myeloproliferative syndromes. Blood 1951:6:372-5.

${ }^{2}$ Bartl R, Frisch B, Burkhardt R. Bone marrow biopsies revisited a new dimension for haematologic malignancies. 2nd ed Basel: Karger, 1985.

${ }^{3}$ Burkhardt R, Bartl R, Jäger $\mathrm{K}$, et al. Chronic myloproliferative disorders (CMPD). Path Res Pract 1984:179:131-86.

${ }^{4}$ Frisch B, Lewis SM, Burkhardt R, Bartl R. Biopsy pathology of bone and bone marrow-biopsy pathology series. London: Chapman and Hall, 1985.

${ }^{5}$ Fialkow PJ. Clonal development and stem cell origin of leukemias and related disorders. In: Gunz FW, Henderson ES, eds. Leukemia. New York: Grune and Stratton, 1983.

${ }^{6}$ Frisch B, Bartl R. Bone marrow biopsies updated-new prospects for clinical diagnostics. In: Essig A, ed. Bibl Haematol, vol 50, Basel: Karger 1984.

${ }^{7}$ Dixon WJ, Brown MB. BMPD-81 biomedical computer programs p-series. System program and statistical development. Berkeley: University of California, 1981.

${ }^{8}$ Harker LA. Megakaryocytic alterations in thrombopoiesis. Blood 1960;24:1014-5.

${ }^{9}$ Burkhardt R, Bartl R, Beil E, et al. Myelofibrosis-osteomyelosclerosis syndrome-review of literature and histomorphology. Advances in the Biosciences 1975:16:9-56.

${ }^{10}$ Georgii A, Vykoupil KF, Thiele J. Chronic megakaryocytic granulocytic myelosis-CMGM. A subtype of chronic myeloid leukemia. Virchows Arch (Pathol Anat) 1980:389:253-68.

${ }^{11}$ Thiele J, Funke S, Holgado S, Choritz H, Heorgii A. Megakaryopoiesis in chronic myeloproliferative diseases. A morphometric evaluation with special emphasis on primary thrombocythemia. Ann Quant Cytol 1984:6:155-67.

${ }^{12}$ Castro-Malaspina H, Moore MAS. Pathophysiological mechanisms operating in the development of myelofibrosis: role of megakaryocytes. Nouv Rev Fr Hematol 1982:24:221-6.

${ }^{13}$ Silverstein MN. Primary thrombocythaemia. In: Williams WJ, Beutler E, Ersley AJ, Lichtman MA, eds. Hematology. New York: McGraw-Hill, 1983:218-26.

Requests for reprints to: Professor R Burkhardt, Abteilung Für Knochenmarksdiagnostik an der Medizinischen Klinik, Innenstadt der Universität München, Zienssenstrasse la, D-8000 München, Federal Republic of Germany. 